\title{
Analysis of Economic Burden of Seasonal Influenza: An Actuarial Based Conceptual Model
}

\author{
S. S. N. Perera \\ Research and Development Center for Mathematical Modeling, Faculty of Science, University of Colombo, 00300 Colombo, Sri Lanka \\ Correspondence should be addressed to S. S. N. Perera; ssnp@maths.cmb.ac.lk
}

Received 15 June 2017; Revised 26 August 2017; Accepted 5 September 2017; Published 11 October 2017

Academic Editor: Winston Garira

Copyright (C) 2017 S. S. N. Perera. This is an open access article distributed under the Creative Commons Attribution License, which permits unrestricted use, distribution, and reproduction in any medium, provided the original work is properly cited.

\begin{abstract}
Analysing the economic burden of the seasonal influenza is highly essential due to the large number of outbreaks in recent years. Mathematical and actuarial models can be considered as management tools to understand the dynamical behavior, predict the risk, and compute it. This study is an attempt to develop conceptual model to investigate the economic burden due to seasonal influenza. The compartment SIS (susceptible-infected-susceptible) model is used to capture the dynamical behavior of influenza. Considering the current investment and future medical care expenditure as premium payment and benefit (claim), respectively, the insurance and actuarial based conceptual model is proposed to model the present economic burden due to the spread of influenza. Simulation is carried out to demonstrate the variation of the present economic burden with respect to model parameters. The sensitivity of the present economic burden is studied with respect to the risk of disease spread. The basic reproduction is used to identify the risk of disease spread. Impact of the seasonality is studied by introducing the seasonally varying infection rate. The proposed model provides theoretical background to investigate the economic burden of seasonal influenza.
\end{abstract}

\section{Introduction}

Infectious diseases such as Dengue, Ebola, SARS, Zika, and various types of influenza are viral infections that are of major public health concern in tropical and subtropical countries [1]. Influenza, generally known as "the flu," is a sessional infectious disease caused by virus. High fever, runny nose, sore throat, muscle pains, headache, cough, and feeling lethargic can be considered as common symptoms. Seasonal influenza can cause mild to severe illness and even death, particularly in some high-risk individuals such as pregnant women, children, very old people, and people with chronic underlying medical conditions such as cancer, diabetes mellitus, and heart diseases. Three different types of influenza viruses generally affect humans, namely, Type $A$, Type B, and Type C. In recent years, the influenza A virus subtypes $\mathrm{H} 1 \mathrm{~N} 1$ and $\mathrm{H} 3 \mathrm{~N} 2$ have been in circulation. In addition, there are two types of $\mathrm{B}$ viruses that are also circulating as seasonal influenza viruses. However, influenza $\mathrm{C}$ may be considered as less of a disease burden [2].

Normally, the influenza virus spreads over a short distance through the air from coughs or sneezes. Human mobility is the main responsible factor for the spread over long distance. Annual influenza outbreaks occur around the world and cause significant morbidity and mortality. Since the virus survives in cold environments with low relative humidity, normally the influenza outbreak can be seen in winter season in the Northern and Southern Hemispheres. Generally, in the tropical and subtropical region outbreaks may occur at any time of the year. However, in most of the tropical countries these peaks of infection are seen mainly during the rainy season [3]. In Sri Lanka, for the last few years, influenza epidemic outbreak has been generally observed during April to June and again in November to January.

From a public health and community point of view, seasonal influenza epidemics spread rapidly and are very difficult to control. Seasonal influenza spreads all over the world as an annual outbreak, making about three to five million severe illness cases and about 250,000 to 500,000 death cases [4]. In the United States approximately, annually, from $5 \%$ to $20 \%$ of the individuals are infected with influenza. Further, the United States health report indicates that about 3,000 to 49,000 influenza-associated deaths occur every year. According to the WHO and health report, in Sri Lanka, 
for the last few years, it has been generally observed that there is high trend concerning the spread of influenza. This epidemics risk causes enormous economic loss and serious adverse events leading to hospitalization. In USA, annual economic burden due to seasonal influenza is approximately more than 1 billion US \$ [3-5]. However, in Sri Lanka the annual economic losses due to this epidemic risk have not yet been addressed.

Due to significant level of outbreaks in and around the world including Sri Lanka during recent years, investigating the economic burden of annual seasonal influenza is motivated. The economic burden can be classified in two ways, namely, individual level which accounts for medical and death expenditures and public level which accounts for the burden due to loss of human days. This study is an attempt to develop the conceptual model to capture economic losses in an individual level due to influenza. However, the proposed tool is only responsible for identifying losses for personal medical care. Classical compartment model is used to describe the spread of disease; model parameters are used to capture the seasonal/climatic impacts. Epidemiological insurance is designed to represent expenditures due to personal medical care and actuarial based computation tool is proposed to compute the economic burden.

This paper is organized in the following way. Classical compartment model, insurance based model, and actuarial tools are presented in Section 2. Mathematical analysis of the model is also presented in Section 2. In Section 3, numerical results and discussion are presented. Finally, conclusion remarks and further steps are highlighted in Section 4.

\section{Mathematical Models}

In this section, first, we introduce the classical compartment deterministic model to study the dynamics of influenza. Secondly, mathematical and actuarial analysis of the epidemic model is discussed and a basic insurance model is constructed.

2.1. Classical Compartment Model. Since influenza virus is constantly changing, people can get infected multiple times throughout their lives. This concept is mathematically modeled by using classical SIS (susceptible $(S)$, infected $(I)$, susceptible) compartments $[6,7]$. Entire population is divided into two compartments, namely, susceptible as previously unexposed to the disease and infected as currently colonized by the virus. Since the objective is to investigate the annual seasonal economic losses due to individual medical care, impacts of the population demography, births, deaths, and migration, are not significant. Hence, omitting such effects, model is developed by considering transition only from compartment $S$ to $I$ and $I$ to $S$. Let $S(t)$ and $I(t)$ denote the number of susceptible and infected ones at time $t$, respectively. The increment of rate of change of infected cases is proportional to the number of susceptible individuals and the number of individuals previously infected. It also noted that such a rate is also proportional to number of transitions from infected to susceptible cases. Taking $b>0$, as a potentially infective contact rate, $p>0$ as the probability of infection per contact, $D>0$ as the average disease duration, and $N$ as a total population, simple SIS model is given by (1a), (1b), and (1c).

$$
\begin{aligned}
& \frac{d S}{d t}=-\frac{p b}{N} S(t) I(t)+\frac{1}{D} I(t), \\
& \frac{d I}{d t}=\frac{p b}{N} S(t) I(t)-\frac{1}{D} I(t),
\end{aligned}
$$

with

$$
\begin{aligned}
S(0) & >0, \\
I(0) & >0, \\
S(t)+I(t) & =N, \quad \forall t \geq 0 .
\end{aligned}
$$

Taking population fraction, $S^{*}(t)=S(t) / N, I^{*}(t)=I(t) / N$, introducing rate of infection, $\beta$, as product of $p$ and $b$ and the rate of recovery, $\gamma=1 / D$, and considering close environment (i.e., $S(t)+I(t)=N, \forall t \geq 0$ ) and dropping $*$, the system (1a), $(1 \mathrm{~b})$, and (1c) reads as $[8,9]$

$$
\frac{d I}{d t}=\beta S(t) I(t)-\gamma I(t),
$$

with initial condition $S(0)>0, I(0)>0$.

2.2. Mathematical Analysis of the Model. Equation (2) can be further simplified and one can obtain an analytical solution given by

$$
\begin{aligned}
\frac{d I}{d t} & =(\beta(1-I(t))-\gamma) I(t) \\
I(t) & =I(0) \int_{0}^{T}(\beta(1-I(t))-\gamma) I(t) d t \\
I(t) & =\frac{(\beta-\gamma)}{\beta+(((\beta-\gamma) / I(0))-\beta) \exp (-(\beta-\gamma) t)} .
\end{aligned}
$$

Equation (2) has two equilibrium points, namely, disease-free equilibrium point $\left(I_{\mathrm{de}}=0\right)$ and the endemic equilibrium point $\left(I_{\mathrm{ee}}=(\beta-\gamma) / \beta\right)$. One can see, using linear stability analysis, disease-free equilibrium point, $I_{\mathrm{de}}$, is locally asymptotically stable if $\beta<\gamma$ and unstable if $\beta>\gamma$, whereas the endemic equilibrium point, $I_{\mathrm{ee}}$, is locally asymptotically stable if $\beta>\gamma$ and unstable if $\beta<\gamma$ [9-11]. The product of the rate of infection, $\beta$, and the average duration of the infectious period, $1 / \gamma$, is the expected number of new infections from one infected individual in a fully susceptible population through the entire duration of the infectious period. This parameter is known as a basic reproduction number, $R_{0}$, and if $R_{0}>1$, an introduced infectious individual leads to more than one infection so the disease spreads in the population. One can easily see that if $R_{0}<1$, the infection in one individual cannot replace itself so the disease dies out. Figure 1 displays variation of infected population fraction with respect to different rate of infection, $\beta$.

The infection rate, $\beta$, depends on the season, demography, and other external factors such as socioeconomic factors, 


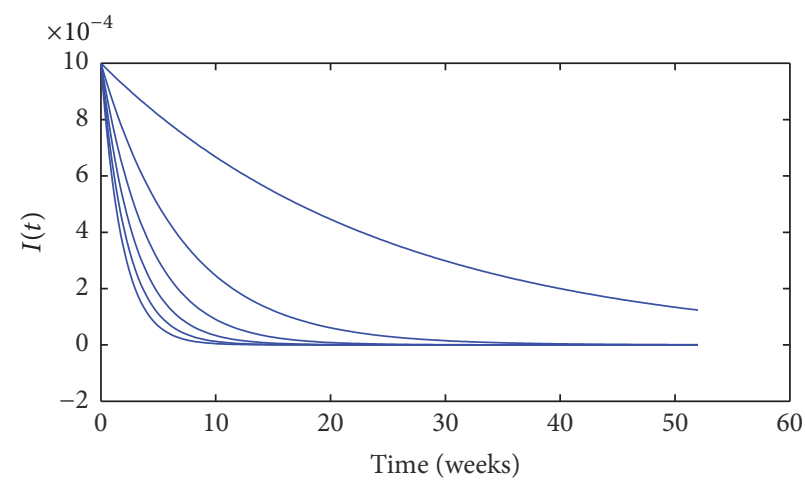

(a)

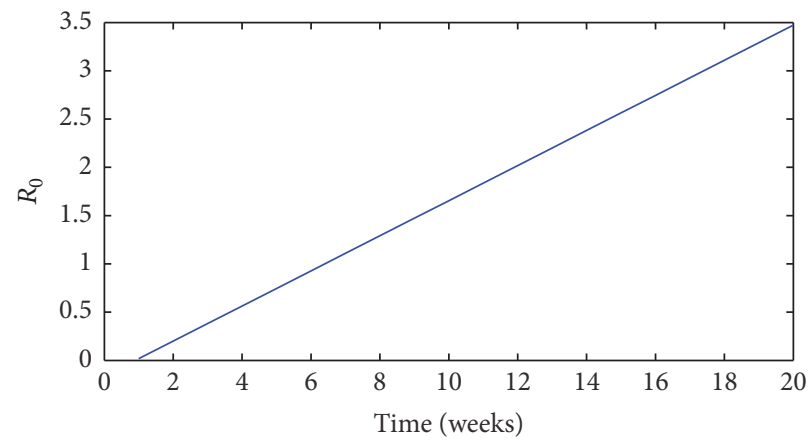

(c)

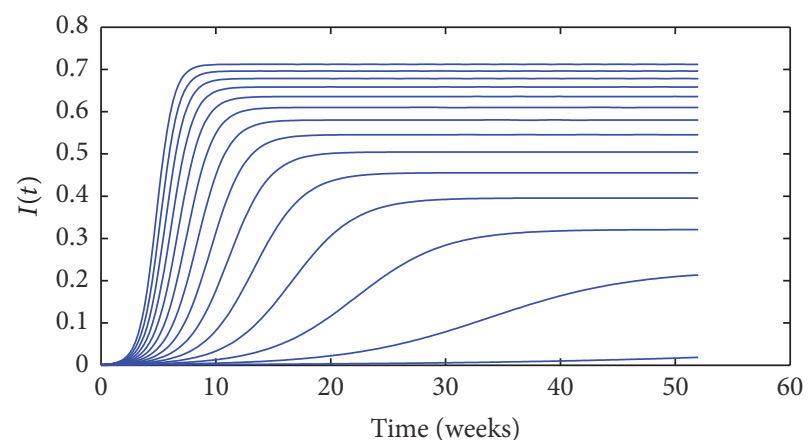

(b)

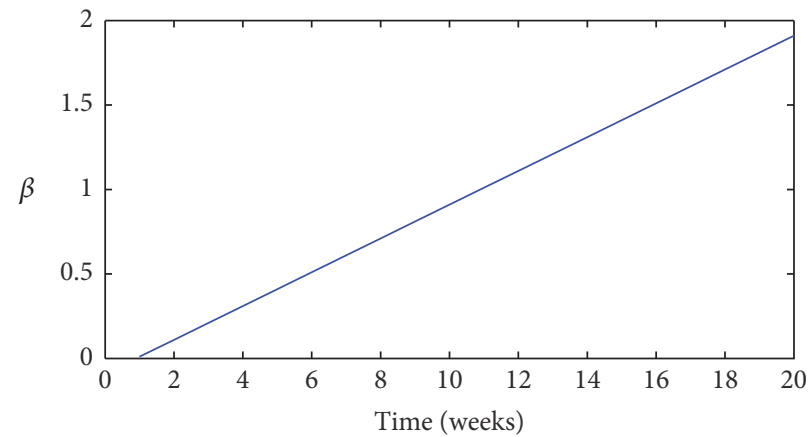

(d)

Figure 1: (a) Variation of infected population fraction when $R_{0}<1$. (b) Variation of infected population fraction when $R_{0}>1$. (c) Corresponding $R_{0}$ variation. (d) Corresponding $\beta$ : this simulation is done, fixing $\gamma=0.55$.

living conditions and life style, and the recovery rate, $\gamma$, which highly depends on the type of population, age group, and other biological factors. Since our objective is to include the seasonality impact, $\beta$ can be chosen to represent seasonal variation. Since the basic reproduction number, $R_{0}$, is defined as the ratio between infection and recovery rates, $R_{0}$ can be considered as the representor of the spread risk of influenza due to external factors. Now $\beta$ may vary periodically due to climatic variations. Mathematically, the seasonal contact rate is generally defined as sinusoidal, such that

$$
\beta(t)=\beta_{0}(1+\omega \cos (2 \pi t)),
$$

where $\omega$ is the amplitude of seasonal variation in transmission and $\beta_{0}$ is the constant infection rate without seasonal impact $[12,13]$. It is understood that sinusoidal forcing of transmission is a crude assumption for influenza. However for simplicity and since our objective is to develop a conceptual model, in this research we incorporate sinusoidal functions.
2.3. Insurance Based Actuarial/Probabilistic Model. In classical SIS model defined in (2), one can see these compartments play similar roles in an insurance model. Susceptible group facing the risk of being infected by influenza form the investment or insurance fund by depositing or investing or paying premiums, to get benefit or return to cover the medical care expenses incurred if infected [14]. During the outbreak of the influenza, the infected policyholders/investors would benefit from the claim (return) payments provided by the insurance/investment fund. Simply speaking the susceptible group make investment to cover future medical expenses. Present investment amount or premium can also be considered as the current economic burden due to the future medical expenditure. Now considering $S(t)$ and $I(t)$ as the probability of an individual being susceptible and infected, respectively, at the time $t$, one can redefine (2) as a probabilistic (an actuarial) model to describe the dynamics of investments versus expenditures.

Considering the expenditure side of the investment (insurance), expected (actuarial) present value of $t$ period unit benefit (claim) payment, $E[B]$, is defined in (5a) [15]:

$$
\begin{aligned}
E[B(t)] & =\int_{0}^{t} \exp (-\delta t) \text { Prob. of an individual being infected at time } t d t \\
& =\int_{0}^{t} \exp (-\delta t) I(t) d t
\end{aligned}
$$


where $\delta>0$ is known as the discounting force of interest. Similarly, one can define the income side of the investment package as the expected (actuarial) present value of $t$ period premium payment (investment), $E[P]$, as in (5c) [15].

$$
\begin{aligned}
E[P(t)] & =\int_{0}^{t} \exp (-\delta t) \text { Prob. of an individual being susceptible at time } t d t \\
& =\int_{0}^{t} \exp (-\delta t) S(t) d t .
\end{aligned}
$$

Using the equivalence principle technique, premium payment/current economic burden, $\pi$, can be obtained [15].

$$
\begin{aligned}
& \pi E \text { [present value of premium] } \\
& =E \text { [present value of future benefit] } .
\end{aligned}
$$

Therefore, $\pi$ can be given in

$$
\pi(t)=\frac{E[B(t)]}{E[P(t)]}=\frac{\int_{0}^{t} \exp (-\delta t) I(t) d t}{\int_{0}^{t} \exp (-\delta t) S(t) d t} .
$$

Expected present premium value given in $(5 \mathrm{c})$ can be further modified.

$$
\begin{aligned}
E & {[P(t)]=\int_{0}^{t} \exp (-\delta t) S(t) d t } \\
& =\int_{0}^{t} S(t) \frac{d}{d t}\left(\frac{\exp (-\delta t)}{-\delta}\right) d t \\
& =\frac{1}{\delta}(S(0)-\exp (-\delta t) S(t))-\frac{1}{\delta} \\
& \cdot \int_{0}^{t} \exp (-\delta t) \frac{d I(t)}{d t} d t=\frac{1}{\delta}(S(0)+I(0) \\
& \left.-\exp (-\delta t)(S(t)+I(t))-\delta \int_{0}^{t} \exp (-\delta t) I(t) d t\right) .
\end{aligned}
$$

Since $S(t)+I(t)=1, \forall t, E[P(t)]$ can be expressed as in

$$
E[P(t)]=\frac{1}{\delta}(1-\exp (-\delta t)-\delta E[B(t)]) .
$$

Now the premium payment/current economic burden, $\pi$, is given in

$$
\pi(t)=\frac{\delta E[B(t)]}{1-\exp (-\delta t)-\delta E[B(t)]} .
$$

The conceptual model to compute the economic burden due to seasonal influenza is given in

$$
\begin{aligned}
\pi(t) & =\frac{\delta E[B(t)]}{1-\exp (-\delta t)-\delta E[B(t)]}, \\
E[B(t)] & =\int_{0}^{t} \exp (-\delta t) I(t) d t, \\
\frac{d I}{d t} & =\beta(1-I(t)) I(t)-\gamma I(t),
\end{aligned}
$$

with initial condition $I(0)>0$.
2.4. Algorithm for Computing the Economic Burden. The system (1la), (11b), and (11c) can be evaluated taking analytical solution given in (3a). By keeping the record of infected population, it is possible to set up the reported infected table and hence the approximated method can be proposed. From the infected table, we obtain a piecewise constant approximation of the continuous function, $I(t)$, and current economic burden, $\pi$, is now given in (12a), (12b), (12c), and (12d).

$$
\begin{aligned}
\widetilde{I}(t) & = \begin{cases}I_{k} ; & k-1<t \leq k \\
0 ; & \text { otherwise. }\end{cases} \\
E[B(t)] & =\int_{0}^{t} \exp (-\delta t) I(t) d t \\
& \approx \int_{0}^{t} \exp (-\delta t) \widetilde{I}(t) d t \\
E[B(t)] & \approx \sum_{k=1}^{m}\left(\frac{\exp (-\delta(k-1))-\exp (-\delta k)}{\delta}\right) I_{k} .
\end{aligned}
$$

Now $\pi$ can be expressed as

$$
\pi(m)=\frac{\vartheta}{1-\exp (-\delta m)-\vartheta},
$$

where

$$
\vartheta=\sum_{k=1}^{m}(\exp (-\delta(k-1))-\exp (-\delta k)) .
$$

\section{Results and Discussion}

Figure 2 displays the variation of the current economic burden (premium) with respect to the contact rate. It is observed that the economic burden rises up when the infection rate, $\beta$, increases. It is clear that, with higher infection rate, the disease spreads out quickly and hence infected fraction is dominant. One can see that current premium is higher than 1 when $\beta$ exceeds 1.8. It means that when infected rate exceeds this threshold value, we need to pay more than one unit in order to have a unit benefit (claim) in future.

Figure 3 shows the variation of the current economic burden (premium) with respect to the infected rate, $\beta$, and recovery rate, $\gamma$. The corresponding variation of basic reproduction number, $R_{0}$, under same domain of infected 




FIGURE 2: Variation of the present economic burden/premium $(\pi)$ with respect to rate of infection $(\beta)$. Here $\gamma=0.55$ and $\delta=0.05$.

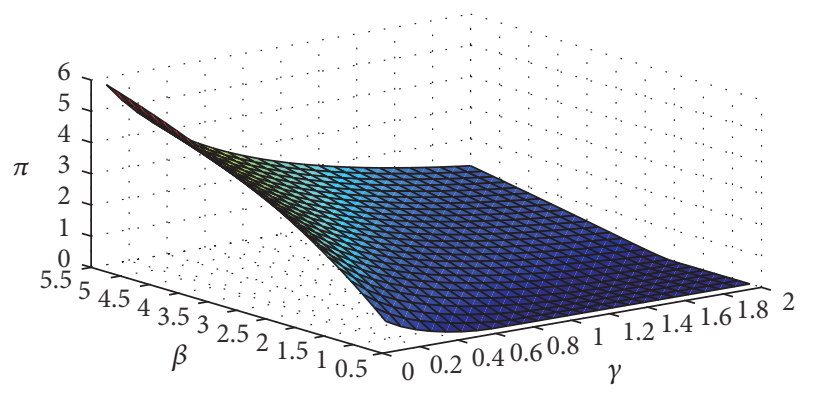

FIGURE 3: Variation of the present economic burden/premium $(\pi)$ with respect to rate of contact $(\beta)$ and $\gamma$. Here $\delta=0.05$.

rate and recovery rate, is given by Figure 4 . Since the basic reproduction number increases significantly, it is understood that the current economic burden also rises. Further, since $R_{0}$ accounts for the spread risk due to various external factors, it is possible to identify the sensitivity of the premium with respect to particular external factor if $R_{0}$ is defined accordingly.

Figure 5 shows the variation of infected population fraction with the constant infection rate and the seasonal infection rate with $\beta_{0}=1.66$. Seasonal variation can be captured via the seasonal infection rate. However, it is noted that, to get realistic situation, we need to identify appropriate parameters in (4).

Figure 6 displays the variation of the current economic burden with respect to time under the infection contact rate and seasonal infection rate. The dynamics of the present economic burden now depends on the amplitude $(\omega)$ of the seasonal variation.

Figure 7 demonstrates the variation of the current economic burden with respect to the discounting force interest, $\delta$, and the basic reproduction number, $R_{0}$. The discounting force of interest is responsible factor for computing the equivalent present amount of future sums. Further, Figure 7 displays the variability of the current economic burden with respect to various external factors as well as future uncertainty too. If $R_{0}$ and $\delta$ are defined appropriately, it is possible to capture the variability of economic burden due to uncertainty of external parameters.

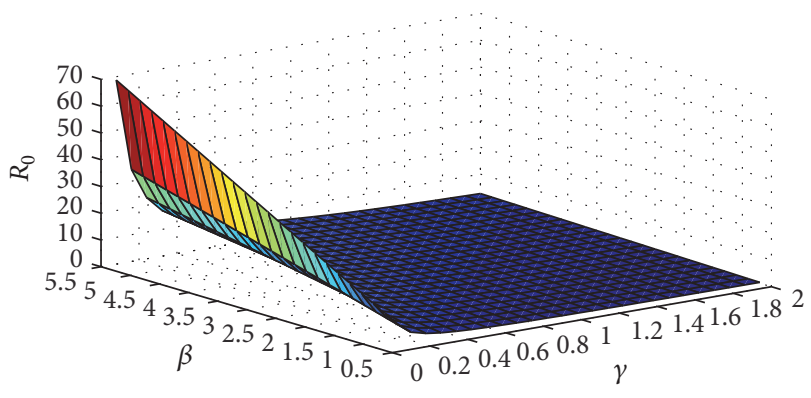

FIGURE 4: Variation of the basic reproduction number $\left(R_{0}\right)$ with respect to rate of contact $(\beta)$ and $\gamma$.

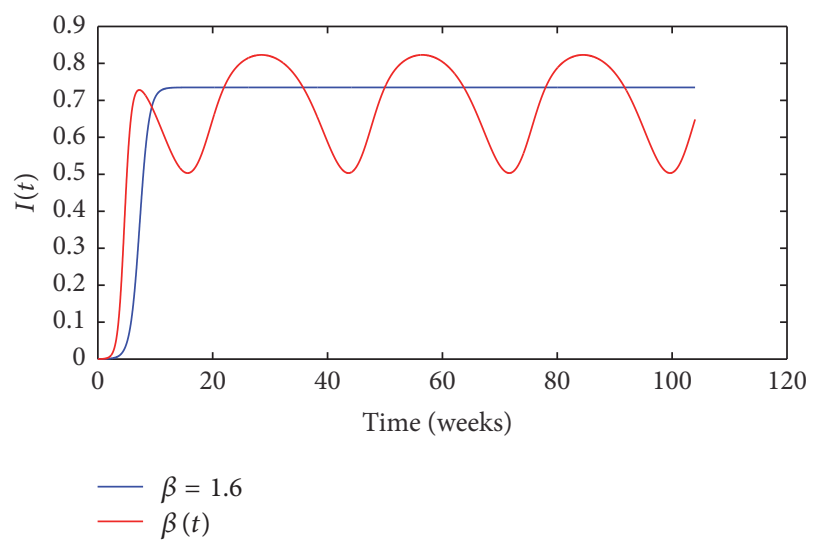

FIGURE 5: Variation of the infected fraction with the constant infection rate, $\beta$ (in blue), and the seasonal infection rate, $\beta(t)$ (in red), defined in (4). Here $\beta_{0}=1.66$ and $\omega=0.28$.



FIGURE 6: Variation of the current economic burden with respect to time under constant infection rate, $\beta$ (in blue), and the seasonal infection rate, $\beta(t)$ (in red), defined in (4). Here $\beta_{0}=1.66$ and $\omega=$ 0.28 .

\section{Conclusions}

The economic burden due to seasonal influenza outbreaks is a critical budgetary problem in many countries due to loss of human hours as well as individual medical care expenditures. Using classical compartment SIS model, the dynamics of influenza is studied. Considering the current 


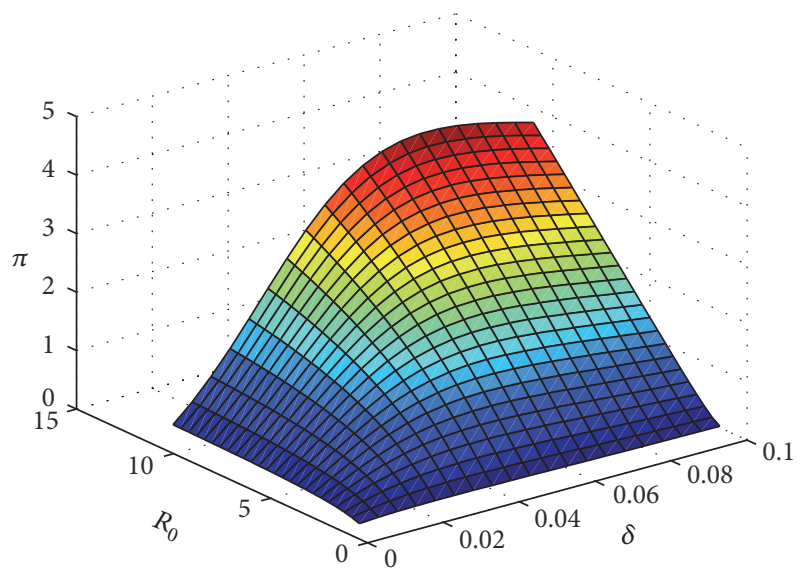

FIGURE 7: Variation of the current economic burden with respect to discounting force interest, $\delta$, and basic reproduction number, $R_{0}$.

investment and future medical care expenditure as premium payment and benefit (claim), respectively, the insurance based conceptual model is proposed to investigate the present economic burden due to the spread of disease. The expected present economic burden is defined as the present investment to cover the future unit medical expenditure. This value is computed using the actuarial based model via equivalence principle. It is observed that the present economic burden depends on model parameters such as the infection rate, $\beta$, and recovery rate, $\gamma$. Since the infection rate highly depends on climatic variation, hence seasonality can be captured by introducing appropriate seasonally dependent function for it. It is also noted that dynamics of the economic burden depends on the amplitude of the seasonality.

This study can be extended to develop feasible premium schemes depending on different seasonal conditions by incorporating with reported infected data. Further, the model can be extended by including indirect cost such as expenditure due to loss of human hours, death, and community expenditures.

\section{Conflicts of Interest}

The author declares that there are no conflicts of interest regarding the publication of this paper.

\section{References}

[1] H. J. Wearing, P. Rohani, and M. J. Keeling, "Appropriate models for the management of infectious diseases," PLOS Medicine, vol. 2, no. 7, pp. 621-627, 2005.

[2] N. J. Cox and K. Subbarao, "Global epidemiology of influenza: past and present," Annual Review of Medicine, vol. 51, pp. 407421, 2000.

[3] Centers for Disease Control and Prevention, "Estimates of deaths associated with seasonal influenza - United States 1976-2007," Morbidity and Mortality Weekly Report, vol. 59, no. 33, pp. 1057-1062, 2010.

[4] K. M. Sullivan, A. S. Monto, and I. M. Longini Jr., "Estimates of the US health impact of influenza," American Journal of Public Health, vol. 83, no. 12, pp. 1712-1716, 1993.
[5] L. Mao, Y. Yang, Y. Qiu, and Y. Yang, "Annual economic impacts of seasonal influenza on US counties: Spatial heterogeneity and patterns," International Journal of Health Geographics, vol. 11, pp. $1-8,2012$.

[6] J. D. Murray, Mathematical Biology, I. Introduction, Springer, 3rd edition, 2002.

[7] D. J. Daley and J. Gani, Epidemic Modeling: An Introduction, Cambridge University Press, 1999.

[8] J. A. Jacquez, Compartmental Analysis in Biology and Medicine, BioMedware, Ann Arbor, MI, USA, 3rd edition, 1996.

[9] H. W. Hethcote, "The mathematics of infectious diseases," SIAM Review, vol. 42, no. 4, pp. 599-653, 2000.

[10] R. M. Anderson and R. M. May, Infectious Diseases of Humans, Oxford Science Publications, 1991.

[11] J. A. Heesterbeek and M. G. Roberts, "Threshold quantities for infectious diseases in periodic environments," Journal of Biological Systems, vol. 3, no. 3, pp. 779-787, 1995.

[12] M. J. Keeling, P. Rohani, and B. T. Grenfell, "Seasonally forced disease dynamics explored as switching between attractors," Physica D: Nonlinear Phenomena, vol. 148, no. 3-4, pp. 317-335, 2001.

[13] N. C. Grassly and C. Fraser, "Seasonal infectious disease epidemiology," Proceedings of the Royal Society B: Biological Sciences, vol. 273, no. 1600, pp. 2541-2550, 2006.

[14] N. Ishimura, D. Komadel, and Y. Yoshizawa, "Risk estimation model on epidemic outbreaks for an insurer," Astin Colloquium, LISBOA, 2016.

[15] N. L. Bowers, H. U. Gerber, J. C. Hickman, D. A. Jones, and C. J. Nesbitt, Actuarial Mathematics, Society of Actuaries, Schaumburg, IL, USA, 1997. 


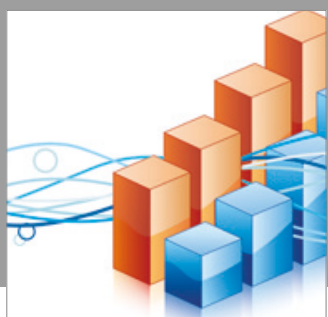

Advances in

Operations Research

vatersals

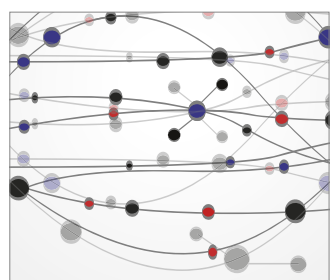

\section{The Scientific} World Journal

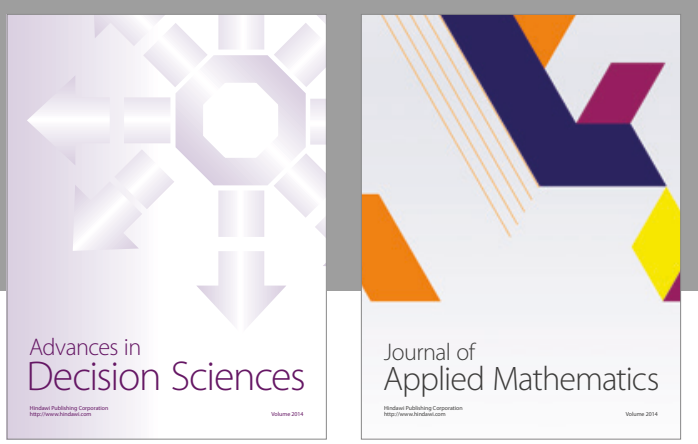

Algebra

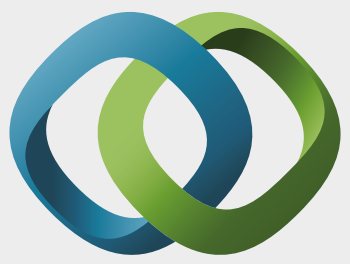

\section{Hindawi}

Submit your manuscripts at

https://www.hindawi.com
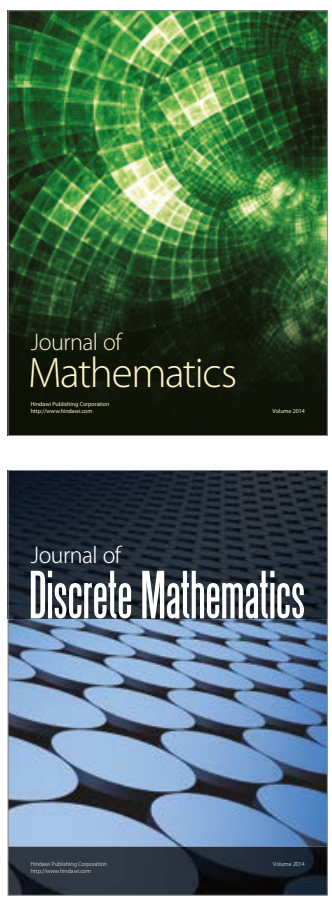



Mathematical Problems in Engineering
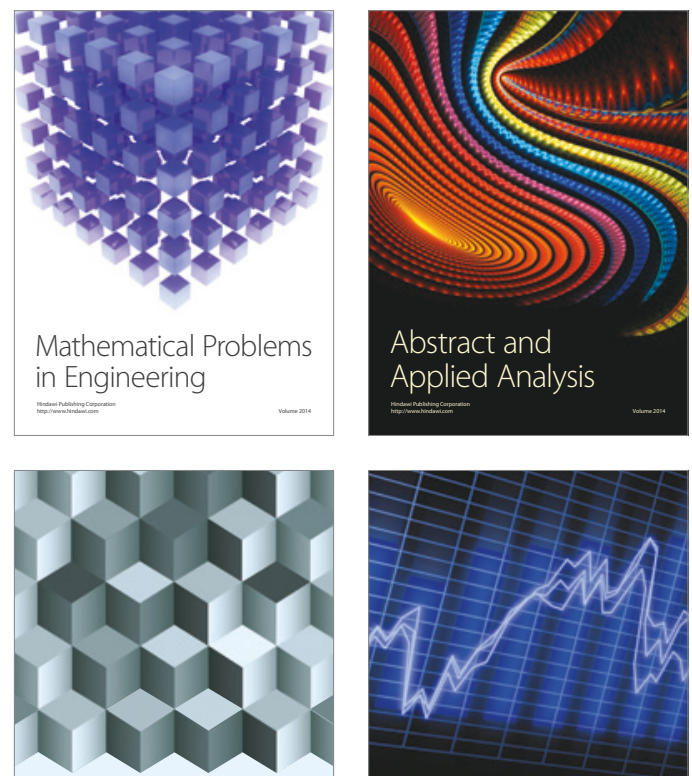

Journal of

Function Spaces



Probability and Statistics
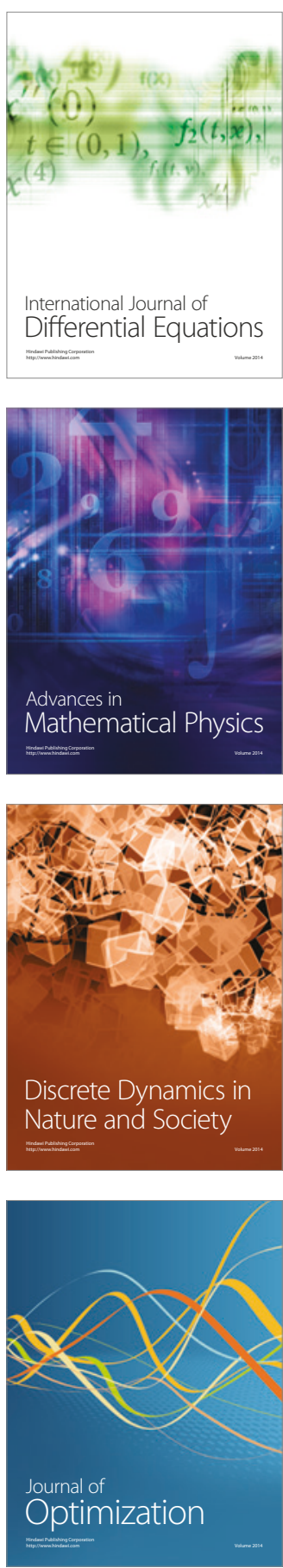